\title{
OCT4B modulates OCT4A expression as ceRNA in tumor cells
}

\author{
DONG LI $^{1 *}$, ZHENG-KAI YANG $^{1 *}$, JING-YI BU ${ }^{1}$, CHUN-YAN XU $^{1}$, HUI SUN $^{1}$, JIE-BING TANG $^{1,3}$, PING LIN ${ }^{1}$, \\ WEN CHENG $^{2}$, NING HUANG ${ }^{1}$, RONG-JUN CUI ${ }^{1}$, XIAO-GUANG YU ${ }^{1}$ and XIU-LAN ZHENG ${ }^{2}$ \\ ${ }^{1}$ Department of Biochemistry and Molecular Biology, Harbin Medical University; \\ Departments of ${ }^{2}$ Ultrasonography and ${ }^{3}$ Clinical Oncology, Harbin Medical University Cancer Hospital, \\ Harbin, Heilongjiang 150081, P.R. China
}

Received December 23, 2014; Accepted February 24, 2015

DOI: $10.3892 /$ or.2015.3862

\begin{abstract}
OCT4 is an essential transcription factor for maintaining the self-renewal and the pluripotency of embryonic stem cells (ESCs). The human OCT4 gene can generate three mRNA isoforms (OCT4A, OCT4B and OCT4B1) by alternative splicing. OCT4A protein is a transcription factor for the stemness of ESCs, while the function of OCT4B isoforms remains unclear. Most types of cancer express a relatively low level of OCT4 protein, particularly the OCT4B isoforms. In the present study, we found that OCT4A and OCT4B mRNA were co-expressed in several types of tumor cell lines and tumor samples, and we demonstrated that OCT4B functioned as a non-coding RNA, modulating OCT4A expression in an miRNA-dependent manner [competing endogenous RNA (ceRNA) regulation] at the post-transcription level in the tumor cell lines. This is the first time that ceRNA regulation was observed among spliced isoforms of one gene, and may pave the way for identification of new targets for cancer treatment.
\end{abstract}

\section{Introduction}

OCT4, also known as POU5F1, is an essential transcription factor of embryonic development, and maintains the pluripotency and self-renewal of embryonic stem cells (ESCs) (1-3). It is a main factor in iPS cell generation (4-6). OCT4 is expressed in several types of cancer and is involved in maintaining cancer stem cell (CSC) properties (7-10), and is associated with the degree of malignancy and the drug-resistance of cancer (11-14).

Correspondence to: Professor Xiao-Guang Yu, Department of Biochemistry and Molecular Biology, Harbin Medical University, Harbin, Heilongjiang 150081, P.R. China

E-mail: xiaoguang_yu@hotmail.com

Professor Xiu-Lan Zheng, Department of Ultrasonography, Harbin Medical University Cancer Hospital, Harbin, Heilongjiang 150081, P.R. China

E-mail: zx12496@163.com

*Contributed equally

Key words: OCT4, ceRNA, miRNA, tumor
There are 3 alternative spliced mRNA isoforms generated by the OCT4 gene: OCT4A, OCT4B and OCT4B1 $(15,16)$. OCT4A mRNA translates into the OCT4A protein which is the canonical OCT4 protein exerting the known function of OCT4 as a transcription factor. OCT4B mRNA generates three protein isoforms through alternative translation initiation: OCT4B-265, OCT4B-190 and OCT4B-164 (17). OCT4B1 mRNA is predicted to generate a potential truncated peptide (18). OCT4A is highly expressed in the nuclei of blastocysts and compacted embryos, whereas OCT4B is found in the cytoplasm of all cells from the four-cell stage onward $(19,20)$. Many studies have focused on the function of OCT4A in contrast to OCT4B. Recently, OCT4B-265 has been reported to be upregulated by genotoxic stress treatment in ES cell lines, and overexpression of OCT4B-265 was found to promote cell apoptosis in a p53-dependent manner (21). In oncology research, most types of cancer express a relatively low level of the OCT4 protein, particularly the OCT4B isoforms (22).

Compared to the low protein level, OCT4A and OCT4B mRNA are co-expressed in several types of tumor cell lines $(22,23)$, yet the OCT4B protein fails to be upregulated after proteasome inhibitor treatment (21). These findings may suggest that in tumor cells OCT4B executes its functions at the RNA level but not as a fully functional protein.

Regulation of gene expression by microRNAs which guide the RNA-induced silencing complex (RISC) to microRNA response elements (MREs) on target transcripts is ubiquitous among mammals, usually resulting in degradation of the transcript or translation inhibition (24). Aberrant expressionfluctuation of a large number of miRNAs has been found in cancer $(25,26)$. Recently, numerous experiments provide support to the hypothesis that RNA molecules that share MREs can regulate each other by competing for microRNA binding, for which the process is termed competing endogenous RNA (ceRNA) regulation (27-32). An OCT4 pseudogene, OCT4-pg4, has been observed to regulate OCT4A expression as an miR-145 sponge in hepatocellular carcinoma (33). Unfortunately, miR-145 is downregulated in most types of cancers, which confines the strength of this regulation (34-36). ceRNA regulation mediated by several pseudogenes has been demonstrated. Yet, whether or not this regulation exists among transcript isoforms and the related mechanism have not been elucidated. Consistent with the co-expression of OCT4A and 
OCT4B, we hypothesized that OCT4B regulates OCT4A as ceRNA.

In the present study, we demonstrated that in the cancer cell lines: i) expression of OCT4B was relatively low at the protein level, yet not at the RNA level; ii) OCT4B regulated OCT4A expression in an miRNA-dependent manner (ceRNA regulation) at the post-transcription level; iii) in addition to miR-145, miR-20a, miR-20b, miR-106a, miR-106b and miR-335 were capable of regulating OCT4. This is the first time that ceRNA regulation has been observed among spliced isoforms, and OCT4B acts as a modulator of OCT4A expression. These findings may pave a way for identification of new targets for cancer treatment.

\section{Materials and methods}

Materials. The primary antibody of OCT4 (ab19857, rabbit pAb, 1:500) was obtained from Abcam (Cambridge, MA, USA), and antibodies against $\beta$-actin (sc-47778, HP-conjugated, 1:500) were purchased from Santa Cruz Biotechnology (Santa Cruz, CA, USA), and HP-conjugated anti-rabbit mouse secondary antibodies were obtained from the Beyotime Institute of Biotechnology (Beyotime, Haimen, China). Three independent anti-Oct4B stealth RNAi siRNAs, Si-1 5-AAGGGATGCAGAGCATCGTGAAAGG-3, Si-2 5-TTTCCATTCGGGATTCAAGAACCTA-3 and $\mathrm{Si}-3$ 5-TAAACACACCAGTTATCAATCTCCC-3, were mixed into an siRNA pool (20 and $6.7 \mu \mathrm{M}$ each). Lipofectamine ${ }^{\circledR} 2000$, RNAiMAX, Opti-MEM ${ }^{\circledR}$ reduced serum media, Stealth ${ }^{\circledR}$ RNAi siRNA and SYBR ${ }^{\circledR}$ Select Master Mix, and NCode ${ }^{\circledR}$ miRNA First-Strand cDNA Synthesis kit were obtained from Invitrogen (Life-Technologies, Carlsbad, CA, USA). X-tremeGENE ${ }^{\circledR} 9$ was purchased from Roche (Roche Applied Science, Basel, Switzerland). RPMI-1640, MEM-EBSS and McCoy's 5A medium and fetal bovine serum (FBS) were obtained from HyClone (Thermo Fisher Scientific, Waltham, MA, USA). MG132 was purchased from Beyotime Institute of Biotechnology. PrimeScript ${ }^{\circledR}$ RT reagent kit with gDNA Eraser was obtained from Takara Bio (Dalian, China). microRNA mimics were obtained from RiboBio (Guangzhou, China).

Plasmid construction. The 3'UTR of OCT4 was amplified from genomic DNA of PA-1 cells and cloned into the EGFP ORF C-terminal of pEGFP-N1 (Clontech, Mountain View, CA, USA) using NotI and XbaI sites, and then subcloned into the psiCHECK-2 vector (Promega, Madison, WI, USA) by the XhoI and NotI sites. The primer sequences were: OCT4 3'UTR-F, 5-TGCCTGCCCTTCTAGGAA-3 and OCT4 3'UTR-R, 5-AAGTGTGTCTATCTACTGTGTCC-3. microRNA-specific mutation psi-CHECK-2 OCT4 3'UTR plasmid $(\Delta 145, \Delta 17, \Delta 335, \Delta 384$ and $\Delta 339)$, were generated using the QuikChange ${ }^{\circledR}$ Site-Directed Mutagenesis kit (Stratagene, Agilent Technologies, Wilmington, DE, USA).

Cell culture and transfection. The human ovarian teratoma cell line PA-1, and the human gastric cancer cell lines MGC803, SGC7901 and NCI-N87, were purchased from the Cell Resource Center, IBMS, CAMS/PUMC. The prostate cancer cell line DU145 was obtained from the American Type Culture Collection (ATCC; Manassas, VA, USA). The colon cancer cell line HCT116 wild-type and Dicer ${ }^{-/}$(HCT116 ${ }^{-/}$) cell line were kindly donated by Professor B. Vogelstein of Johns Hopkins University. PA-1 cells were maintained in MEM-EBSS medium, HCT116 and HCT116-/. were maintained in McCoy's 5A medium and the other cell lines were maintained in RPMI-1640 medium. All culture media were supplemented with $10 \% \mathrm{FBS}$ and $100 \mathrm{U} / \mathrm{ml}$ penicillin/streptomycin (both from HyClone, Thermo Fisher Scientific), at $37^{\circ} \mathrm{C}$ in a humidified atmosphere $\left(5 \% \mathrm{CO}_{2} / 95 \%\right.$ air $)$.

OCT4 3'UTR was cloned into the pEGFP-N1 vector and marked as pEGFP-Oct4 3'UTR. DNA transfection was performed using X-tremeGENE ${ }^{\circledR} 9$ when cells reached $80 \%$ confluency. For siRNA/miRNA mimic transfection (100 nM), Lipofectamine ${ }^{\circledR}$ RNAiMAX was utilized, when cells reached a 50\% confluency. Lipofectamine ${ }^{\circledR} 2000$ was used for reporter gene plasmid and miRNA mimic co-transfection. Transfections were performed according to the manufacturer's recommendations in 6-well plates.

Cell proliferation analysis. Eight hours post-transfection, the cells were trypsinized, resuspended and seeded in four separate 24-well plates at a final density of 10,000/well. Starting from the following day (day 0), one plate/day was washed once with PBS, fixed in 4\% paraformaldehyde solution for $10 \mathrm{~min}$ at room temperature, and then stained with $0.05 \%$ crystal violet for $30 \mathrm{~min}$. After lysis with methanol, the absorbance was read at $592 \mathrm{~nm}$ on a microplate spectrophotometer (SpectraMax ${ }^{\circledR}$ M3; Molecular Devices, Sunnyvale, CA, USA).

RNA extraction and real-time PCR. For real-time PCR analyses, total RNA was extracted from the cells using TRIzol ${ }^{\circledR}$ (Ambion, Life-Technologies) reagent as per the manufacturer's instructions. Several types of RNA were donated by Dr Wang (HMU). Clearance of DNA contamination in RNA and cDNA synthesis was performed using the PrimeScript ${ }^{\circledR}$ RT reagent kit with gDNA Eraser according to the manufacturer's instructions. MicroRNA reverse transcription was performed using NCode ${ }^{\circledR}$ miRNA First-Strand cDNA synthesis kit according to the manufacturer's instructions. Real-time PCR was subsequently performed using the ABI-7500 system employing SYBR ${ }^{\circledR}$ Select Master Mix. Primer sequences were: OCT4A F, 5-CCCCTGGTGCCGTGAA-3 and OCT4A R, 5-GCAAATTGCTCGAGTTCTTTCTG-3; OCT4B F, 5-CAG GGAATGGGTGAATGAC-3 and OCT4B R, 5-AGGCAG AAGACTTGTAAGAAC-3; GAPDH F, 5-GAGTCAACG GATTTGGTCGT-3 and GAPDH R, 5-GACAAGCTTCCCGT TCTCAG-3.

\section{Luciferase assays}

Validation of miRNA regulation by OCT4. HCT116 cells were seeded $24 \mathrm{~h}$ before transfection at a density of 50,000 cells/well in 24-well plates. psiCHECK-2 OCT4 3'UTR WT vector (500 ng) or miR-145/miR-335/miR-384/miR-339/miR-17a site mutation vector was co-transfected with corresponding miRNA mimics with Lipofectamine ${ }^{\circledR} 2000$ according to the manufacturer's instructions.

Detection of OCT4A/B reciprocal regulation. HCT116 and PA- 1 cells were seeded $24 \mathrm{~h}$ before transfection at a density of 50,000 or 60,000 cells/well in 24-well plates. psiCHECK-2 
OCT4 3'UTR WT vector (500 ng) was co-transfected with the corresponding $800 \mathrm{ng}$ pEGFP-Oct4 3'UTR/pEGFP empty vector with Lipofectamine 2000 according to the manufacturer's instructions.

In all cases, firefly luciferase gene in psiCHECK-2 was used as a normalization control for transfection efficiency. At 48/72 h after transfection, firefly and Renilla luciferase activities were measured consecutively with the Dual-Luciferase reporter assay system using a luminometer (both from Promega).

Western blotting assay. Whole-cell lysate preparation and western blot analysis were carried out as previously described (37).

Bisulfite sequencing analysis. Bisulfite sequencing PCR (BS-PCR) was performed with genomic DNA from the PA-1, HCT116, HCT116 ${ }^{-1}$, MGC803 and DU145 cells with EpiTect Fast LyseAll Bisulfite kit (Qiagen, Hilden, Germany). PCR reactions were performed using EpiTaq HS (Takara Bio) with Nest PCR primers: OCT4 distal enhancer (DE) outer F, 5-AG GAGTTATTAGGAAAATGGGTAGTAG-3 and OCT4 DE outer R, 5-TACCTTCTAAAAAAATAAATATCCC-3; OCT4 DE inner F, 5-ATTTGTTTTTTGGGTAGTTAAAGGT-3 and OCT4 DE inner R, 5-CCAACTATCTTCATCTTAATAACA TCC-3.

The second PCR products were subcloned using pMD-19 $\mathrm{T}$ vector (Takara Bio) according to the manufacturer's protocol, and individual clones were subsequently sequenced (Sangon Biotech, Shanghai, China). Clones were only accepted if there was at least $90 \%$ cytosine conversion, and all possible clonalities were excluded based on criteria from the BiQ Analyzer software (Max Planck Society, Munich, Germany). At least 10 replicates were performed for each of the selected regions in each cell line.

Co-expression of OCT4A and OCT4B analysis. For qRT-PCR assessment, data were analyzed using the $\Delta \Delta \mathrm{Ct}$ method; for co-expression in invasive breast cancer, prostate and colon cancer samples, processed and normalized expression data were downloaded from the TCGA database, and then analyzed with RSEM (RNA-Seq by Expectation-Maximization).

Statistical analysis. Each experiment was repeated at least in triplicate. Statistical analyses (Student's t-test) were performed using Microsoft Excel. $p<0.05$ ( $<<0.05, p<0.01, p<0.001$ as indicated in the figures) was considered to indicate a statistically significant result, and results are expressed as mean \pm SD .

\section{Results}

Ubiquitous expression of OCT4A and OCT4B mRNA but not protein in the tumor cell lines. To measure expression of OCT4 in the tumor cells, we used qRT-PCR and western blotting to detect the RNA and protein expression levels of OCT4 (Fig. 1A and B). Consistent with a previous study, OCT4 protein was absent in most of the somatic cancer cell lines (22). At the RNA level, we found that OCT4A and OCT4B expression were correlated to each other (Fig. 1A). In order to confirm whether co-expression of OCT4A/B exists
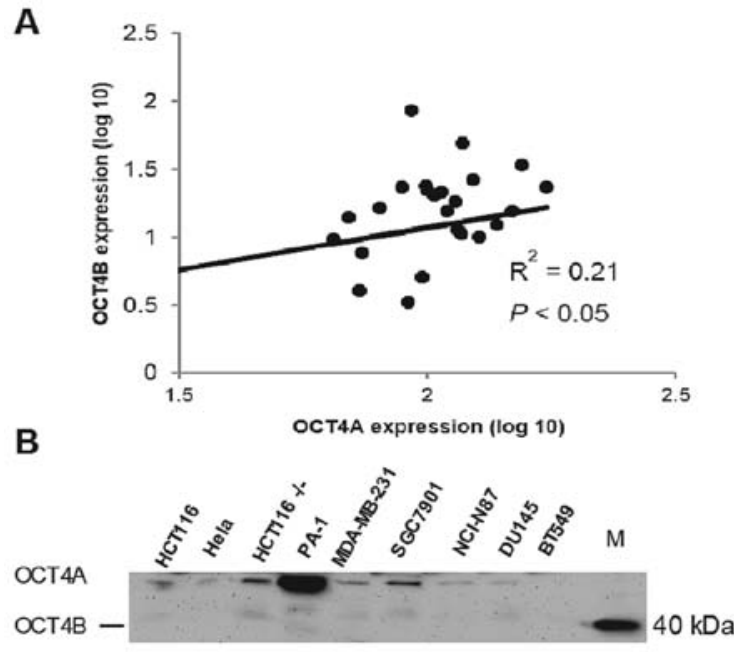

C

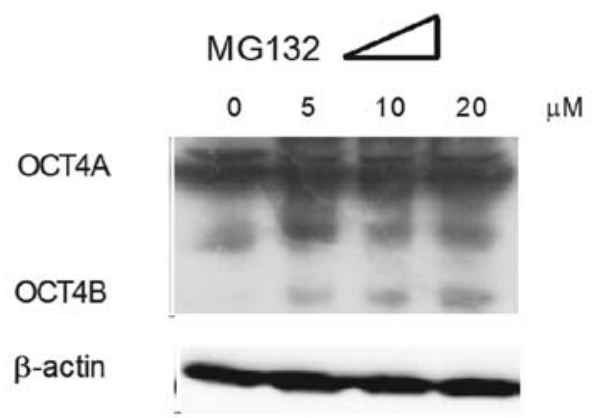

Figure 1. OCT4A and OCT4B expression in the different types of cancer cell lines. (A) RNA co-expression of OCT4A and OCT4B in various cancer cell lines, as determined by qRT-PCR. (B) OCT4 protein expression in the different cancer cell lines. (C) OCT4A and OCT4B protein expression in the PA-1 cell line, $10 \mathrm{~h}$ post-treatment with a proteasome inhibitor (MG132).

in the tumor samples, we analyzed expression data from the TCGA database. After removing samples that did not express OCT4A or OCT4B (no raw sequencing read), expression of OCT4A/B was found to be correlated in invasive breast, prostate and colon cancer samples (Fig. 2A-C). To eliminate any bias generated by our analysis method, an OCT4 pseudogene transcript, POU5F1B, acting as a valid microRNA-145 sponge that regulates OCT4A expression was also analyzed (33). The results indicated that POU5F1B also showed a positive correlation to OCT4A expression in the aforementioned cancers (Fig. 2D-F).

We further exposed PA-1 (a teratoma cell line) to MG132, a proteasome inhibitor, which has been proven to upregulate OCT4B in these cells (21). As expected, OCT4B was upregulated following a 10-h treatment with MG132 in a concentration-dependent manner (Fig. 1C). Yet, MG132 was unable to upregulate OCT4B protein in the colon cancer cell lines HCT116 and HCT15 and in the prostate cancer cell lines DU145 and PC-3 (data not shown).

OCT4B modulates OCT4A expression as ceRNA. To confirm our hypothesis, we utilized anti-OCT4B siRNA knockdown of OCT4B or overexpression of OCT4B 3'UTR (as for OCT4 $3^{\prime}$ UTR, due to the fact that OCT4B 3'UTR and OCT4A 

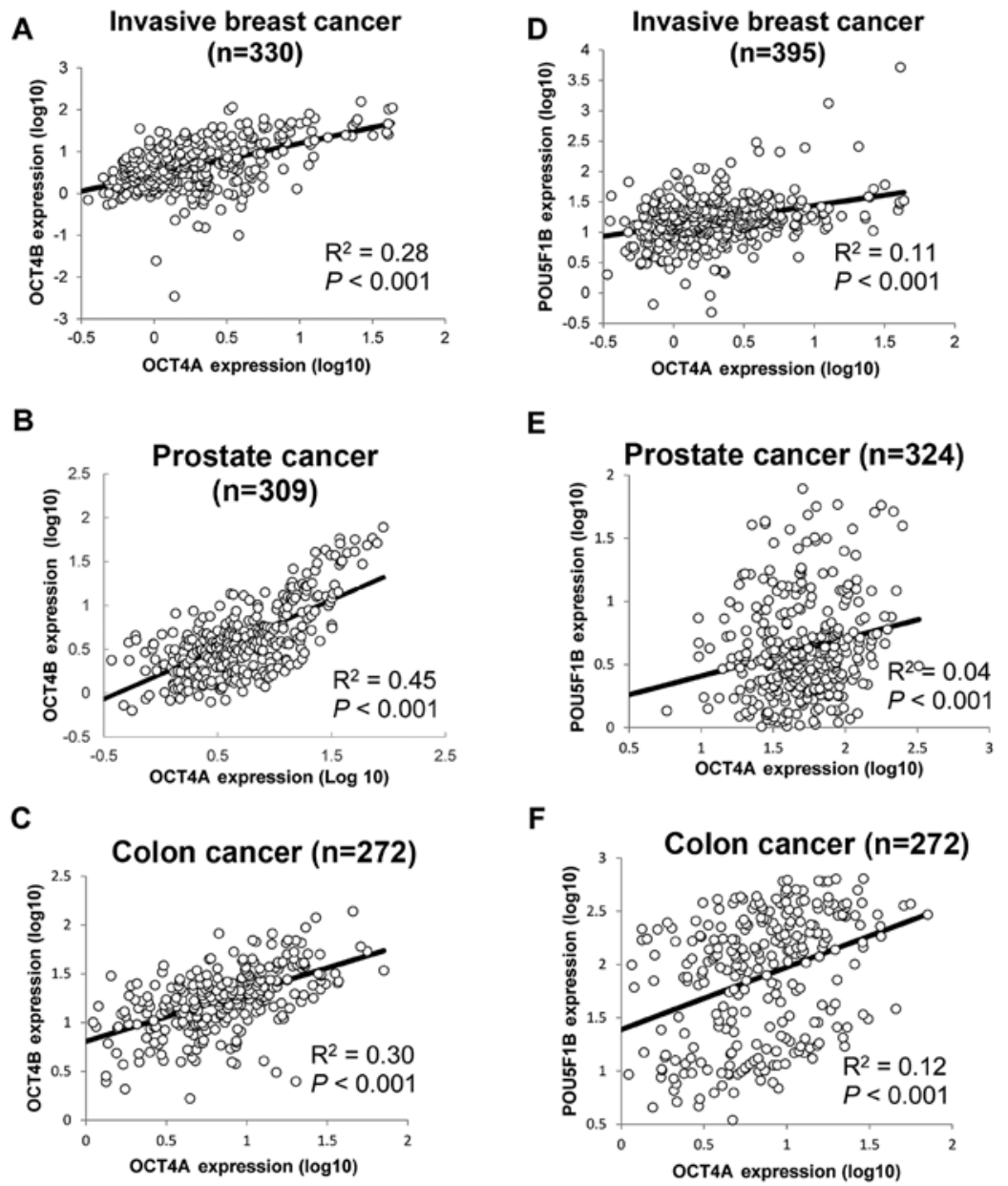

Figure 2. Co-expression of OCT4A, OCT4B and POU5F1B in different types of cancer from the TCGA database. Co-expression of OCT4A and OCT4B in invasive (A) breast, (B) prostate and (C) colon cancer. Co-expression of OCT4A and POU5F1B in invasive (D) breast, (E) prostate and (F) colon cancer.

3'UTR are identical). In the PA-1 cells, anti-OCT4B siRNA inhibited OCT4B and OCT4A at the RNA and protein levels. Overexpression of OCT4 3'UTR increased OCT4A and OCT4B protein expression (Fig. 3A and C). Since miR-145 has been confirmed to regulate SOX2, we assessed whether SOX2 expression was affected. SOX2 protein also fluctuated in the process with the same tendency as OCT4 (Fig. 3C).

To ascertain whether this observed effect is dependent upon OCT4 3'UTR, we constructed a chimeric luciferase plasmid tagged with the OCT4 3'UTR (Luc-OCT4 3'UTR). In the PA-1 and HCT116 cells, overexpression of OCT4 3'UTR enhanced Luc-OCT4 3'UTR activity, but in Dicer-deficit HCT116 cells (HCT116 ${ }^{-/}$cells do not express the majority of mature miRNAs), Luc-OCT4 3'UTR activity was not affected, indicating that mature microRNAs are essential for the regulation (Fig. 3B). Furthermore, overexpression of OCT4 3'UTR increased OCT4A protein expression in the HCT116 cells, but not in the Dicer-deficit HCT116 ${ }^{-/}$cells (Fig. 3D and E), and MG132 was unable to elevate OCT4B protein expression (Fig. 3D).

microRNA pool regulates OCT4 expression. To verify the microRNAs that mediate OCT4A/B ceRNA modulation, we used the miRanda algorithm to predict OCT4-targeting miRNAs and detected their expression levels; apart from
miR-106a, predicted miRNAs, miR-145, miR-20a, miR-20b, miR-384, miR-106b, miR-335 and miR-339 were expressed at a higher level in the PA-1 and HCT116 cells, in contrast to the levels in the HCT116 ${ }^{-/-}$cells (Fig. 4A). miR-145 has been reported to regulate OCT4 expression (38). In the HCT116 cells, expression of miR-145, miR-335, miR-20a, miR-20b, miR-106a and miR-106b significantly reduced Luc-OCT4 3'UTR activity according to mutation construct, respectively. Overexpression of miR-339 and miR-384 did not obviously affect Luc-OCT4 3'UTR activity (Fig. 4B). Since miR-106a/b and $\mathrm{miR}-20 \mathrm{a} / \mathrm{b}$ belong to the miR-17 family, we further tested the effect of miR-17a expression on Luc-OCT4 3'UTR activity, and the results demonstrated a negative effect (Fig. 4B).

Consistent with the luciferase results, overexpression of miR-145, miR-335, miR-20a, miR-20b, miR-106a and miR$106 \mathrm{~b}$ caused a significant downregulation of OCT4 protein in the HCT116 cells (Fig. 4C).

Cell proliferation is regulated by $O C T 4 A / B$ ceRNA interaction. We next investigated the biological functions of ceRNA regulation among OCT4A/B. Previous studies indicated that suppression of OCT4A inhibited cell proliferation, and suppression of OCT4B sensitized A549 cells to cisplatin $(39,40)$. To evaluate the effects of anti-OCT4B siRNA or overexpression of OCT4 3'UTR on cell proliferation, a 
A

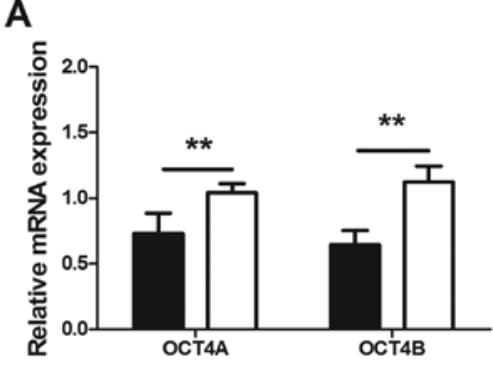

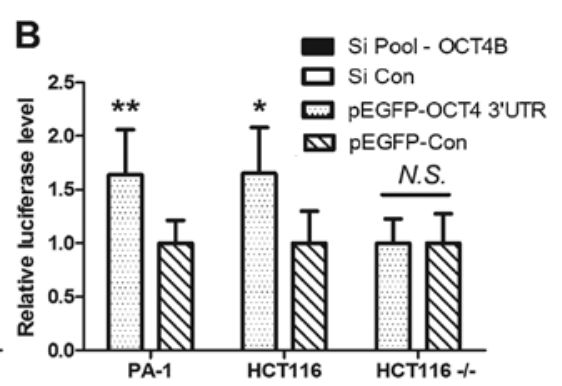
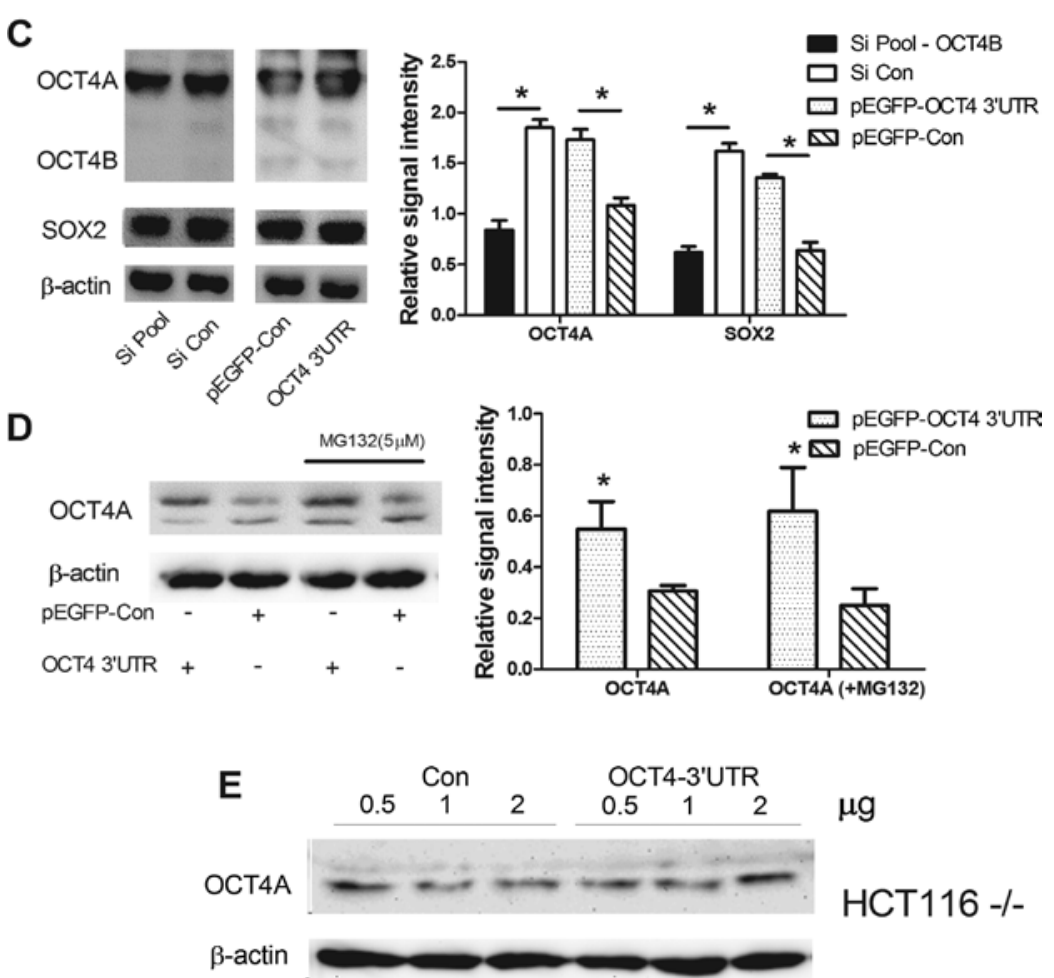

Figure 3. OCT4B modulates OCT4A expression as ceRNA. (A) RNA expression of OCT4A/B post-transfection with anti-OCT4B siRNA or overexpression of OCT4 3'UTR. (B) Luciferase activity in PA-1, HCT116 and HCT116 ${ }^{--}$cells cotransfected with pEGFP-OCT4 3'UTR and a luciferase-OCT4 3'UTR reporter plasmid. (C) OCT4 and SOX2 protein expression in the PA-1 cells after knockdown of OCT4B or overexpression of OCT4 3'UTR. (D) OCT4 protein expression in the HCT116 cells after overexpression of OCT4 3'UTR, without or with a 10-h MG132 treatment. Mean \pm SD; n $>3$; ${ }^{*}$ p $<0.05 ;{ }^{* *}$ p $<0.01 ;$ N.S., not significant. (E) OCT4 protein expression in the HCT116 $6^{-/}$cells following forced expression of OCT4 3'UTR with various vector volumes in transfection.

cell proliferation assay was performed. The results revealed that in the PA-1 cells knockdown of OCT4B significantly inhibited cell growth (Fig. 5A). In contrast, overexpression of OCT4 3'UTR in the PA-1 and HCT116 cells promoted cell proliferation (Fig. 5B and C), but not in the $\mathrm{HCT}_{116^{-/}}$cell line (Fig. 5D).

OCT4 promoter is highly methylated in somatic cancer cell lines. To elucidate the underlying mechanisms involved in the low level of expression of OCT4A/B in tumor cells, the epigenetic factors were investigated.

Methylation of a distal enhancer region of the OCT4 promoter was reported to be associated with OCT4 expression (22). We utilized bisulfite sequencing to detect methylation of this region in the teratoma cell line PA- 1 and in 4 different somatic cancer cell lines. The results revealed that in the HCT116, HCT116 $6^{-1}$, MGC803 and DU145 cell lines, all 5 CpG sites showed a relative high level of methylation, in contrast to the PA-1 cells (Fig. 6). Yet, notably, DNA methylation inhibitor, decitabine, failed to enhance the expression of OCT4A/B in the HCT116 and DU145 cells (data not shown).

\section{Discussion}

OCT4 is an essential transcription factor of embryonic development, involving maintenance of the pluripotency and self-renewal of embryonic stem cells (ESCs) (1-3), and is a main factor in iPS cell generation (4-6). OCT4 is expressed in several types of cancer facilitating the maintenance of cancer stem cell (CSCs) properties (7-10), and is associated with the degree of malignancy and the drug-resistance character of cancer (11-14).

Three alternative spliced mRNA isoforms are generated by the OCT4 gene: OCT4A, OCT4B and OCT4B1 (Fig. 7A) $(15,16)$. OCT4A mRNA translates into the OCT4A protein which is the canonical OCT4 protein exerting the known function of OCT4 as a transcription factor. OCT4B mRNA generates three protein isoforms through alternative transla- 
A

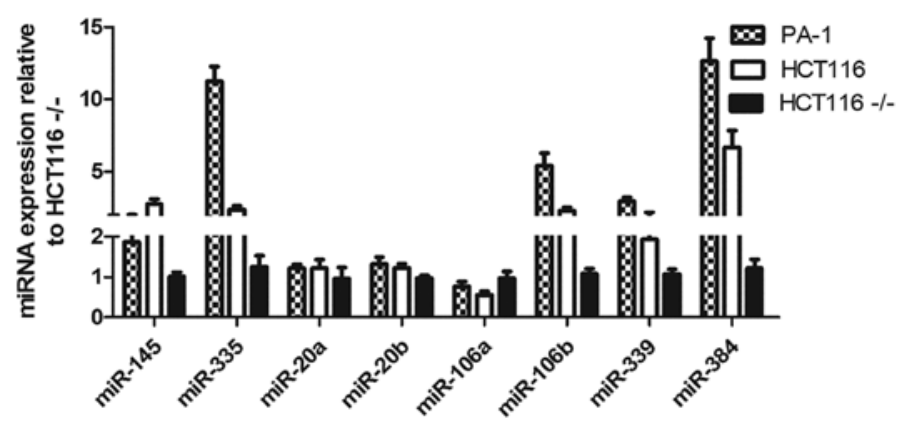

B

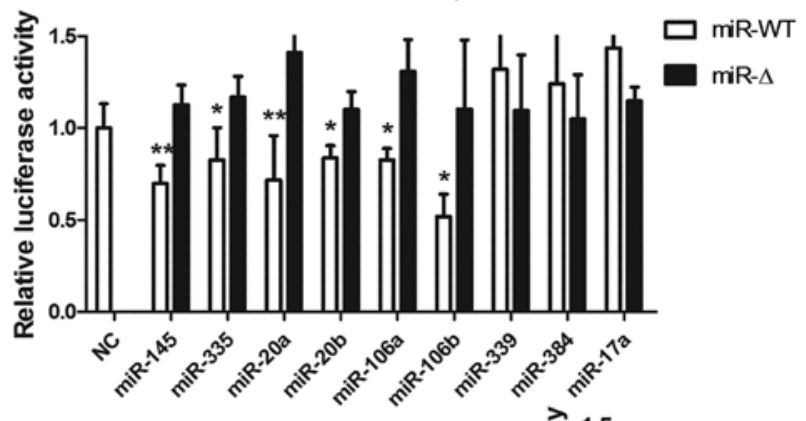

C
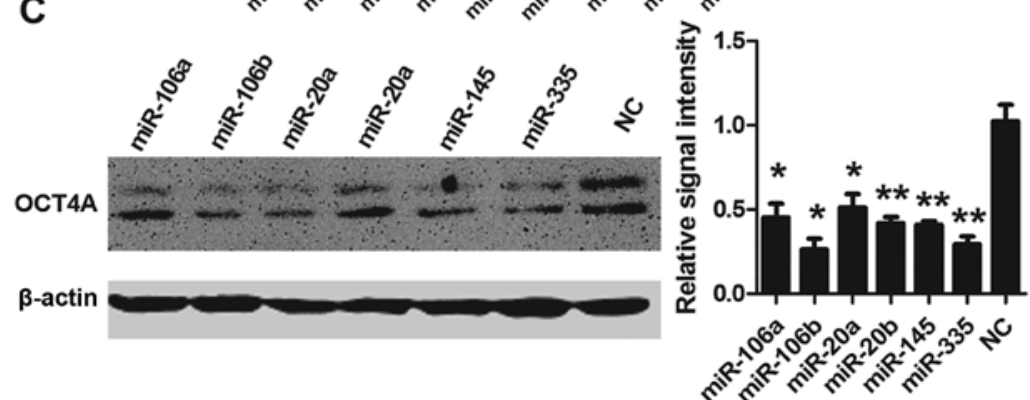

Figure 4. Expression of OCT4 is regulated by microRNAs. (A) Expression of predicted OCT4-targeting microRNAs in the PA-1, HCT116 and HCT116 ${ }^{-/}$cell lines. (B) Luciferase activity in the HCT116 cells cotransfected with predicted OCT4-targeting microRNAs and luciferase-OCT4 3'UTR reporter plasmid. (C) OCT4 protein expression in the HCT116 cells transfected with reporter gene assay-validated OCT4-targeting microRNAs. Mean \pm SD; $n>3$; " $p<0.05$; *** $<0.01$.
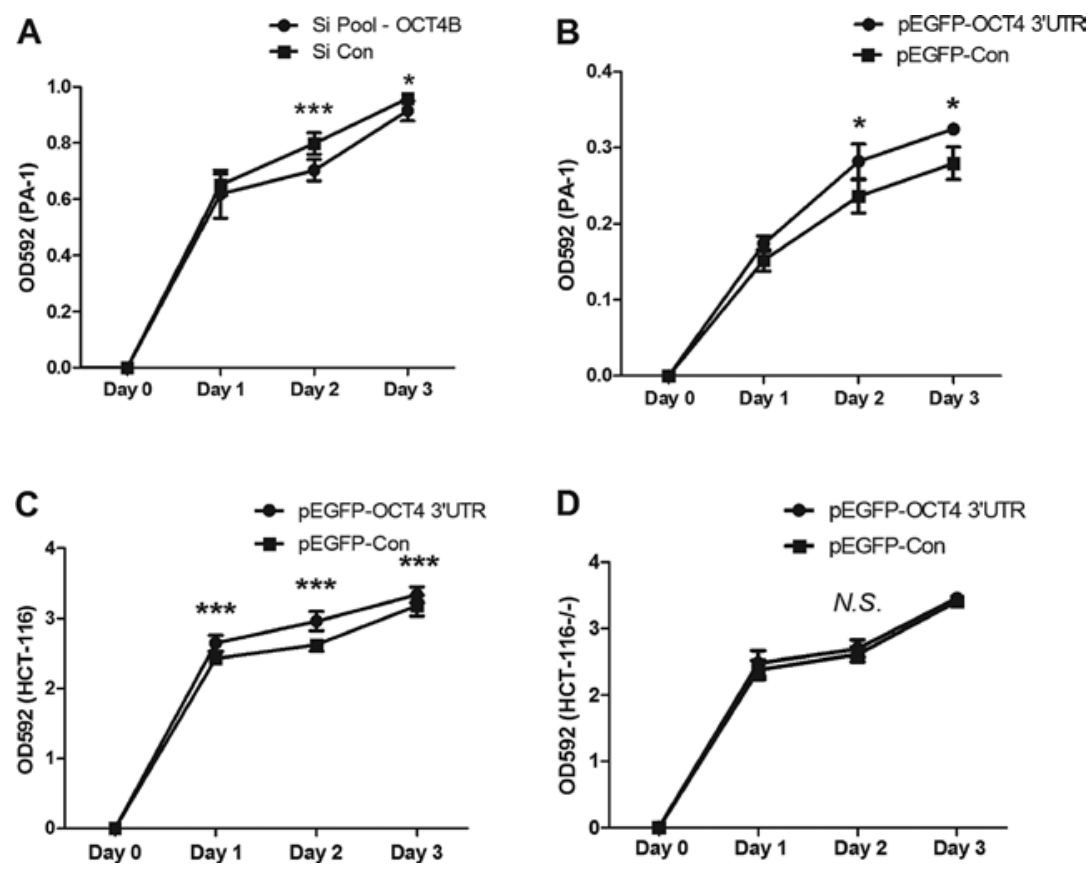

Figure 5. Cell proliferation is regulated by OCT4A/B ceRNA interaction. (A and B) Cell proliferation curve of PA-1 cells transfected with anti-OCT4B siRNA or overexpression of OCT4 3'UTR. (C) Cell proliferation curve of HCT116 cells transfected with pEGFP-OCT4 3'UTR. (D) Cell proliferation curve of $\mathrm{HCT}_{116}{ }^{-/}$cells transfected with pEGFP-OCT4 3'UTR. Mean $\pm \mathrm{SD} ; \mathrm{n}>3 ;{ }^{*} \mathrm{p}<0.05 ;{ }^{* * *} \mathrm{p}<0.001 ;$ N.S., not significant. 


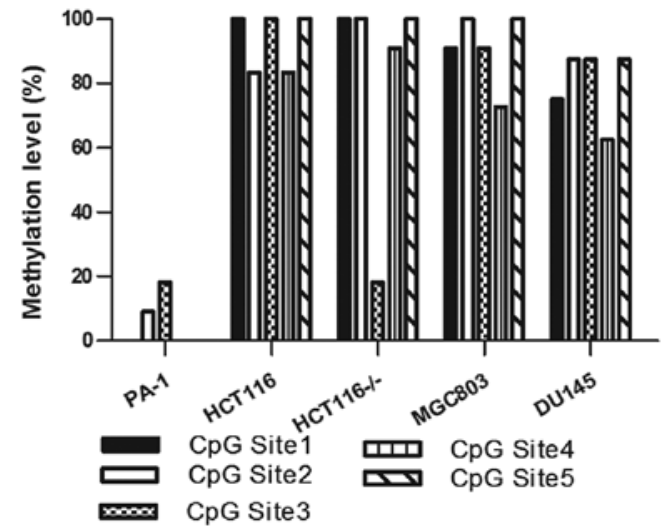

Figure 6. Methylation of the distal enhancer region of the OCT4 promoter.
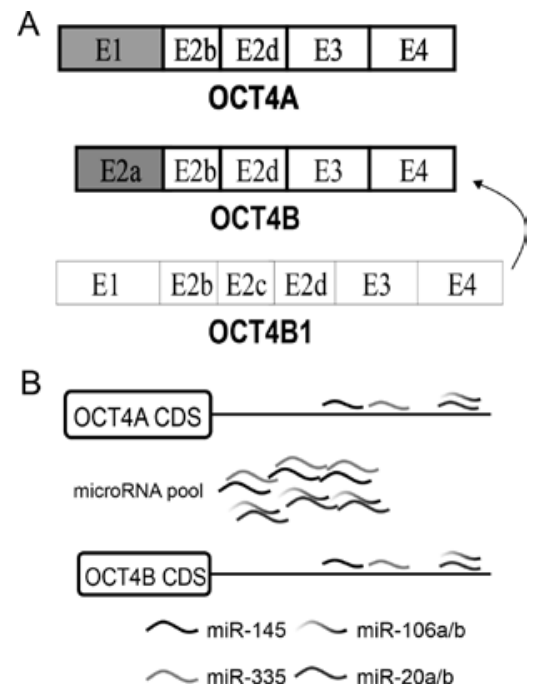

Figure 7. Alternative splicing and microRNA regulation of OCT4. (A) Schematic structure of human OCT4 gene mRNA and protein products. Three mRNA isoforms OCT4A, OCT4B, and OCT4B1 are generated from the OCT4 gene. (B) Schematic outline of the miRNA-mediated mutual regulation of OCT4A and OCT4B.

tion initiation: OCT4B-265, OCT4B-190 and OCT4B-164 (17). OCT4B1 mRNA has been predicted to generate a potential truncated peptide and could convert to OCT4B (18).

Based on previous research and our results, expression of OCT4, particularly OCT4B, was relative lower in the tumor cell lines when compared to embryonic cells. At the protein level, OCT4B was undetectable by western blotting (Fig. 1B). (21,22). Evidence in ES cells shows that OCT4B proteins are degraded quickly by the proteasome pathway, and MG132 blocks the degradation of OCT4B $(21,41)$. In several tumor cell lines, MG132 was unable to upregulate expression of OCT4B, whereas OCT4A and OCT4B RNA expression were correlated with each other in the tumor cell lines and tumor samples (Figs. 1A and 2A-C). Thus, we hypothesized that OCT4B may function as a non-coding RNA, yet not a coding RNA in tumors.

Recently, the boundary between coding RNAs and non-coding RNAs has become obscure (42). A coding transcript may exert a non-coding function by competive binding with miRNAs and act as a trans-modulator of other gene expression for the same miRNA-target $(27,29,30)$. This ceRNA regulation has been found between gene/pseudogene and among diverse gene transcripts. Here, in the present study, for the first time, we found a ceRNA relationship between 2 alternative spliced transcripts of one gene; OCT4B regulated OCT4A expression by competive binding with microRNAs (Figs. 3 and 7). During the process, we also found a group of miRNAs that target OCT4; agreeing with most research, miRNA targetpredicting algorithms have a low precision, compared to the experimental detection methods (Fig. 4B) (43). Our results also support that manipulation of OCT4B expression may alter cell proliferation, consistent with a previous study on OCT4B (39). We believe that the reported anti-apoptotic property of OCT4B is based on its impact on OCT4A, acting as ceRNA. Our subsequent research will focus on this aspect. In the course of overexpression of OCT4 3'UTR, SOX2 was also upregulated. Yet, due to the absence of SOX2 expression in the HCT116-/ cells, we could not ascertain whether or not the underlying mechanisms are dependent upon the competive binding of miR-145 by OCT4 3'UTR.

Confusingly, anti-OCT4B siRNA was unable to downregulate OCT4A in the HCT116 cells (data not shown). One explanation may be that knockdown of the low expression of OCT4B was not enough to perturb the abundance of OCT4targeting miRNAs. Abundance of miRNAs and endogenous target sites may play a key role in ceRNA regulation (44).

DNA methylation has been reported to be associated with gene expression; hypermethylated $\mathrm{CpG}$ site at $\mathrm{CpG}$ islands in the gene promoter is associated with gene transcription (45-47). Methylation of a distal enhancer region of the OCT4 promoter was reported to be related to OCT4 expression (Fig. 6) (22). According to our results, DNA methylation inhibitor, decitabine, was unable to increase the expression of OCTA or OCT4B in the HCT116 and DU145 cells. This demonstrated that more complicated epigenetic modifications are involved, apart from DNA methylation (48-52).

In conclusion, we demonstrated that: i) expression of OCT4B is low at the protein level, yet not at the RNA level; ii) OCT4B modulates OCT4A expression via an miRNAdependent manner (ceRNA regulation) at the post-transcription level; iii) in addition to miR-145, miR-20a, miR-20b, miR-106a, miR-106b and miR-335 are capable of targeting OCT4. This is the first time that ceRNA regulation was observed among spliced isoforms, and OCT4B acts as a modulator of OCT4A expression.

\section{Acknowledgements}

We thank the members of the laboratory of Dr Yu for critical assessment of the manuscript, and H.B. Bao, H.Y.Liu, N. Wang, X.C. Wang, L.L. Yang, Y. Chen and W.J. Li. (Harbin Medical University) for discussions and the several types of cell RNA. We thank B. Vogelstein for the DICER ${ }^{-/-}$cells. Co-expression results were entirely or partly based upon data generated by the TCGA Research Network: http://cancergenome.nih.gov/. D.L. was supported by a Challenge Cup Undergraduate Innovation Award of Heilongjiang Province and Spring Thunder Project of Harbin Medical University. The present study was supported by a grant from Harbin Science and Technology Bureau (grant no. RC2014XK004031) to X.L.Z., a grant from 
the National Youth Natural Science Foundation of China (grant no. 81101942) to P.L., and a grant from Undergraduate Enterprise Program of Heilongjiang Province to D.L., in part.

\section{References}

1. Nichols J,Zevnik B, Anastassiadis K, Niwa H, Klewe-Nebenius D, Chambers I, Schöler H and Smith A: Formation of pluripotent stem cells in the mammalian embryo depends on the POU transcription factor Oct4. Cell 95: 379-391, 1998.

2. Niwa H, Miyazaki J and Smith AG: Quantitative expression of Oct-3/4 defines differentiation, dedifferentiation or self-renewal of ES cells. Nat Genet 24: 372-376, 2000.

3. Boyer LA, Lee TI, Cole MF, et al: Core transcriptional regulatory circuitry in human embryonic stem cells. Cell 122: 947-956, 2005.

4. Hay DC, Sutherland L, Clark J and Burdon T: Oct-4 knockdown induces similar patterns of endoderm and trophoblast differentiation markers in human and mouse embryonic stem cells. Stem Cells 22: 225-235, 2004.

5. Takahashi K, Tanabe K, Ohnuki M, Narita M, Ichisaka T, Tomoda K and Yamanaka S: Induction of pluripotent stem cells from adult human fibroblasts by defined factors. Cell 131: 861-872, 2007.

6. Park IH, Zhao R, West JA, Yabuuchi A, Huo H, Ince TA, Lerou PH, Lensch MW and Daley GQ: Reprogramming of human somatic cells to pluripotency with defined factors. Nature 451: 141-146, 2008.

7. Kumar SM, Liu S, Lu H, Zhang H, Zhang PJ, Gimotty PA, Guerra M, Guo W and Xu X: Acquired cancer stem cell phenotypes through Oct4-mediated dedifferentiation. Oncogene 31: 4898-4911, 2012.

8. Reers S, Pfannerstill AC, Maushagen R, Pries R and Wollenberg B: Stem cell profiling in head and neck cancer reveals an Oct-4 expressing subpopulation with properties of chemoresistance. Oral Oncol 50: 155-162, 2014.

9. Chiou SH, Yu CC, Huang CY, Lin SC, Liu CJ, Tsai TH, Chou SH, Chien CS, Ku HH and Lo JF: Positive correlations of Oct-4 and Nanog in oral cancer stem-like cells and high-grade oral squamous cell carcinoma. Clin Cancer Res 14: 4085-4095, 2008.

10. Chen YC, Hsu HS, Chen YW, et al: Oct-4 expression maintained cancer stem-like properties in lung cancer-derived CD133-positive cells. PLoS One 3: e2637, 2008.

11. Atlasi Y, Mowla SJ, Ziaee SA and Bahrami AR: OCT-4, an embryonic stem cell marker, is highly expressed in bladder cancer. Int J Cancer 120: 1598-1602, 2007.

12. Linn DE, Yang X, Sun F, Xie Y, Chen H, Jiang R, Chen H, Chumsri S, Burger AM and Qiu Y: A role for OCT4 in tumor initiation of drug-resistant prostate cancer cells. Genes Cancer 1: 908-916, 2010

13. de Resende MF, Chinen LT, Vieira S, Jampietro J, da Fonseca FP, Vassallo J, Campos LC, Guimarães GC, Soares FA and Rocha RM: Prognostication of OCT4 isoform expression in prostate cancer. Tumour Biol 34: 2665-2673, 2013.

14. Wen K, Fu Z, Wu X, Feng J, Chen W and Qian J: Oct-4 is required for an antiapoptotic behavior of chemoresistant colorectal cancer cells enriched for cancer stem cells: Effects associated with STAT3/Survivin. Cancer Lett 333: 56-65, 2013.

15. Takeda J, Seino S and Bell GI: Human Oct3 gene family: cDNA sequences, alternative splicing, gene organization, chromosomal location, and expression at low levels in adult tissues. Nucleic Acids Res 20: 4613-4620, 1992.

16. Asadi MH, Mowla SJ, Fathi F, Aleyasin A, Asadzadeh J and Atlasi Y: OCT4B1, a novel spliced variant of OCT4, is highly expressed in gastric cancer and acts as an antiapoptotic factor. Int J Cancer 128: 2645-2652, 2011.

17. Wang X, Zhao Y, Xiao Z, et al: Alternative translation of OCT4 by an internal ribosome entry site and its novel function in stress response. Stem Cells 27: 1265-1275, 2009.

18. Atlasi Y, Mowla SJ, Ziaee SA, Gokhale PJ and Andrews PW: OCT4 spliced variants are differentially expressed in human pluripotent and nonpluripotent cells. Stem Cells 26: 3068-3074, 2008.

19. Cauffman G, Liebaers I, Van Steirteghem A and Van de Velde H: POU5F1 isoforms show different expression patterns in human embryonic stem cells and preimplantation embryos. Stem Cells 24: 2685-2691, 2006.
20. Cauffman G, Van de Velde H, Liebaers I and Van Steirteghem A: Oct-4 mRNA and protein expression during human preimplantation development. Mol Hum Reprod 11: 173-181, 2005.

21. Gao Y, Wei J, Han J, Wang X, Su G, Zhao Y, Chen B, Xiao Z, Cao J and Dai J: The novel function of OCT4B isoform-265 in genotoxic stress. Stem Cells 30: 665-672, 2012

22. Cantz T, Key G, Bleidissel M, Gentile L, Han DW, Brenne A and Schöler h: Absence of OCT4 expression in somatic tumor cell lines. Stem Cells 26: 692-697, 2008.

23. Zhao S, Yuan Q, Hao H, et al: Expression of OCT4 pseudogenes in human tumours: Lessons from glioma and breast carcinoma. J Pathol 223: 672-682, 2011.

24. Bartel DP: MicroRNAs: Target recognition and regulatory functions. Cell 136: 215-233, 2009.

25. Mendell JT and Olson EN: MicroRNAs in stress signaling and human disease. Cell 148: 1172-1187, 2012.

26. Ebert MS and Sharp PA: Roles for microRNAs in conferring robustness to biological processes. Cell 149: 515-524, 2012.

27. Tay Y, Rinn J and Pandolfi PP: The multilayered complexity of ceRNA crosstalk and competition. Nature 505: 344-352, 2014.

28. Tay Y, Kats L, Salmena L, et al: Coding-independent regulation of the tumor suppressor PTEN by competing endogenous mRNAs. Cell 147: 344-357, 2011.

29. Salmena L, Poliseno L, Tay Y, Kats L and Pandolfi PP: A ceRNA hypothesis: The Rosetta Stone of a hidden RNA language? Cell 146: 353-358, 2011

30. Karreth FA, Tay Y, Perna D, et al: In vivo identification of tumorsuppressive PTEN ceRNAs in an oncogenic BRAF-induced mouse model of melanoma. Cell 147: 382-395, 2011.

31. Miranda KC, Huynh T, Tay Y, Ang YS, Tam WL, Thomson AM, Lim B and Rigoutsos I: A pattern-based method for the identification of microRNA binding sites and their corresponding heteroduplexes. Cell 126: 1203-1217, 2006.

32. Fang L, Du WW, Yang X, et al: Versican 3'-untranslated region (3'-UTR) functions as a ceRNA in inducing the development of hepatocellular carcinoma by regulating miRNA activity. FASEB J 27: 907-919, 2013.

33. Wang L, Guo ZY, Zhang R, et al: Pseudogene OCT4-pg4 functions as a natural micro RNA sponge to regulate OCT4 expression by competing for miR-145 in hepatocellular carcinoma. Carcinogenesis 34: 1773-1781, 2013.

34. Yoshino H, Enokida H, Itesako T, Kojima S, Kinoshita T, Tatarano S, Chiyomaru T, Nakagawa M and Seki N: Tumorsuppressive microRNA-143/145 cluster targets hexokinase-2 in renal cell carcinoma. Cancer Sci 104: 1567-1574, 2013.

35. Iio A, Takagi T, Miki K, Naoe T, Nakayama A and Akao Y: DDX6 post-transcriptionally down-regulates miR-143/145 expression through host gene NCR143/145 in cancer cells. Biochim Biophys Acta 1829: 1102-1110, 2013.

36. Kojima S, Enokida H, Yoshino H, et al: The tumor-suppressive microRNA-143/145 cluster inhibits cell migration and invasion by targeting GOLM1 in prostate cancer. J Hum Genet 59: 78-87, 2014.

37. Lin F, Lin $\mathrm{P}, \mathrm{Z}$ hao $\mathrm{D}$, et al: Sox 2 targets cyclinE, 27 and survivin to regulate androgen-independent human prostate cancer cell proliferation and apoptosis. Cell Prolif 45: 207-216, 2012.

38. Xu N, Papagiannakopoulos T, Pan G, Thomson JA and Kosik KS: MicroRNA-145 regulates OCT4, SOX2, and KLF4 and represses pluripotency in human embryonic stem cells. Cell 137: 647-658, 2009.

39. Cortes-Dericks L, Yazd EF, Mowla SJ, Schmid RA and Karoubi G: Suppression of OCT4B enhances sensitivity of lung adenocarcinoma A549 cells to cisplatin via increased apoptosis. Anticancer Res 33: 5365-5373, 2013.

40. Lin H, Sun LH, Han W, et al: Knockdown of OCT4 suppresses the growth and invasion of pancreatic cancer cells through inhibition of the AKT pathway. Mol Med Rep 10: 1335-1342, 2014.

41. Gao Y, Wang X, Han J, Xiao Z, Chen B, Su G and Dai J: The novel $O C T 4$ spliced variant $O C T 4 B 1$ can generate three protein isoforms by alternative splicing into OCT4B. J Genet Genomics 37: 461-465, 2010.

42. Smith JE, Alvarez-Dominguez JR, Kline N, Huynh NJ, Geisler S, $\mathrm{Hu} \mathrm{W}$, Coller J and Baker KE: Translation of small open reading frames within unannotated RNA transcripts in Saccharomyces cerevisiae. Cell Rep 7: 1858-1866, 2014.

43. Li J, Zhang Y, Wang Y, Zhang C, Wang Q, Shi X, Li C, Zhang R and $\mathrm{Li} \mathrm{X}$ : Functional combination strategy for prioritization of human miRNA target. Gene 533: 132-141, 2014. 
44. Denzler R, Agarwal V, Stefano J, Bartel DP and Stoffel M: Assessing the ceRNA hypothesis with quantitative measurements of miRNA and target abundance. Mol Cell 54: 766-776, 2014.

45. Ramsahoye BH, Davies CS and Mills KI: DNA methylation: Biology and significance. Blood Rev 10: 249-261, 1996.

46. Leonhardt H, Rahn HP and Cardoso MC: Functional links between nuclear structure, gene expression, DNA replication, and methylation. Crit Rev Eukaryot Gene Expr 9: 345-351, 1999.

47. Ruvinsky A: Basics of gametic imprinting. J Anim Sci 77 (Suppl 2): S228-S237, 1999.

48. Amente S, Lania L and Majello B: The histone LSD1 demethylase in stemness and cancer transcription programs. Biochim Biophys Acta 1829: 981-986, 2013.
49. Chase A and Cross NC: Aberrations of EZH2 in cancer. Clin Cancer Res 17: 2613-2618, 2011.

50. Kellner S and Kikyo N: Transcriptional regulation of the Oct4 gene, a master gene for pluripotency. Histol Histopathol 25: 405-412, 2010.

51. Lee $\mathrm{YH}$ and $\mathrm{Wu} \mathrm{Q}$ : Chromatin regulation landscape of embryonic stem cell identity. Biosci Rep 31: 77-86, 2011.

52. Das S and Levasseur D: Transcriptional regulatory mechanisms that govern embryonic stem cell fate. Methods Mol Biol 1029: 191-203, 2013. 